Revue d'histoire de l'enfance " irrégulière »

Le Temps de l'histoire

$8 \mid 2006$

Le corps du délinquant

\title{
Les « corps perdus » de Mettray
}

Myriam Bendhif-Syllas

\section{(2) OpenEdition}

\section{Journals}

Édition électronique

URL : http://journals.openedition.org/rhei/377

DOI : $10.4000 /$ rhei.377

ISBN : 978-2-7535-1646-5

ISSN : 1777-540X

Éditeur

Presses universitaires de Rennes

Édition imprimée

Date de publication : 15 novembre 2006

Pagination : 133-148

ISSN : 1287-2431

Référence électronique

Myriam Bendhif-Syllas, «Les « corps perdus » de Mettray », Revue d'histoire de l'enfance «irrégulière » [En ligne], 8 | 2006, mis en ligne le 01 décembre 2008, consulté le 04 décembre 2020. URL : http:// journals.openedition.org/rhei/377 ; DOI : https://doi.org/10.4000/rhei.377

(c) PUR 


\section{Les « corps perdus " de Mettray}

\section{Myriam Bendhif-Syllas $^{(1)}$}

Dans le cadre de mon travail de doctorat sur les ouvres romanesques de Jean Genet, j'ai découvert ce qu'on appelait alors un " bagne d'enfants ", la colonie pénitentiaire de Mettray. Genet aborde plus particulièrement cette époque de sa vie dans Miracle de la rose. Abandonné à la naissance par sa mère, orphelin de père, il fut confié à une famille du Morvan où il vécut jusqu'à son adolescence. Son enfance semble se dérouler sans problème. Il se distingue par ses résultats scolaires exceptionnels et son goût pour la lecture. S’il échappe au sort de ses condisciples de l'Assistance - il ne sera pas valet de ferme -, il est destiné à devenir apprenti typographe dans la prestigieuse école professionnelle D'Alembert. Mais cette décision ne lui convient pas. On peut supposer qu'il aurait souhaité poursuivre des études secondaires. ${ }^{(2)}$ Commence alors une période où se succèdent fugues, vagabondages, petits vols. Il entre à Mettray en juillet 1926 à l'âge de 16 ans. Il quittera la colonie en mars 1929 lors de son engagement militaire, devançant de deux ans sa sortie.

Il faut noter d'emblée le statut particulier de ce texte. Il ne s'agit pas d'un livre de souvenirs ou d'un témoignage, au sens strict. C'est avant tout une œuvre littéraire, conçue comme telle par Jean Genet dont c'est le deuxième ouvrage. L'écrivain y évoque, quoique avec une certaine distance, ses souvenirs de colon, ainsi que sa vie de prisonnier adulte dans une maison d'arrêt centrale. Le récit comporte de nombreux passages qui apportent des informations sur les réalités de la colonie et qui restituent le quotidien avec une grande précision. ${ }^{(3)}$ Or, le récit de type autobiographique alterne et se confond même avec des passages de pure fiction. Ainsi, non seulement Genet efface de son récit les raisons de son placement à Mettray, mais il prétend être entré à Mettray, dans un premier passage, parce qu'il aurait crevé l'œil gauche d'un gamin, ${ }^{(4)}$ puis, dans un deuxième, parce qu'il aurait participé à une

\author{
(1) Enseignante en
}

lettres modernes, Myriam

Bendhif-Syllas prépare

une thèse sur l'influence

de $\grave{A}$ la recherche du temps

perdu de Marcel Proust

sur l'œuvre romanesque

de Jean Genet. Elle a

publié « Le "bagne d'en-

fants" ou l' "engagement

poétique" de Jean Genet

dans Miracle de la rose"

dans la revue Entre'Actes de mai 2005 et s'intéresse

aux représentations de

l'enfance et de l'homo-

sexualité dans la littérature

des XIXème et XXème siècles.

(2) Jablonka Ivan, Les vérités inavouables de Jean Genet, Paris, Seuil, 2004, voir en particulier p. $98-123$.

Myriam Bendhif-Syllas / p. 133 à 148 
(3) Les biographes attestent par ailleurs la véracité de la plupart de ses dires. Voir Albert Dichy et Pascal Fouché, Jean Genet. Essai de chronologie I9I01944, Bibliothèque Jean Genet, Bibliothèque de littérature française contemporaine de l'université Paris 7, Paris, 1988, 294 p. ; E. Moraly, Jean Genet, la vie écrite, Paris, éditions de la Différence, 1988, 353 p. ; Edmund White, Jean Genet, (traduit par P. Delamare), Paris, NRF, Gallimard, 1993, 686 p., voir en particulier p. 74-98.

(4) Jean Genet, Miracle de la rose [1946], Folio, Gallimard, 1977, p. 280-281.

(5) Ibid., p. 332-334.

(6) Raoul Léger, $L a$ colonie agricole et pénitentiaire de Mettray. Souvenirs d'un colon 1922-1927, Paris, L'Harmattan, 1997, révolte sanglante ${ }^{(5)}$ qui n'est attestée nulle part. Cet ouvrage au statut ambigu, mêlant fiction et récit autobiographique, rend la définition de son genre littéraire très délicate, voire impossible. Il m’a semblé nécessaire de confronter le récit de Genet à d'autres témoignages d'anciens colons, afin de tenter de distinguer plus clairement les différentes strates de cette œuvre complexe.

J'ai eu recours à plusieurs ouvrages concernant le sujet des bagnes d'enfants, ainsi qu'aux récits de Raoul Léger et de Jean-Guy Le Dano ${ }^{(6)}$ qui se présentent tous deux comme des témoins désireux de donner une vision juste et vraie de la colonie et de ce qu'ils y ont vécu. Raoul Léger fut colon à Mettray de 1922 à 1927. Abandonné par son père, il intègre à 7 ans l'Assistance publique. Après plusieurs placements et autant de fugues, il entre à Mettray dans la section des plus jeunes : il a 11 ans. Son témoignage, recueilli par Jacques Bourquin, raconte avec simplicité dans un style proche de l'oralité, ses souvenirs bons et mauvais de la colonie. On note le souci d'être précis et de nuancer les autres récits qui ont été faits sur le sujet. Quant à Jean-Guy Le Dano, il offre le récit de son "tour de France " des maisons de redressement et des maisons d'arrêt centrales dans La Mouscaille. Il précise en ouverture du livre qu'il ne s'agit pas d'" un roman, [que tous] les faits relatés sont strictement authentiques ».(7) $\mathrm{Il}$ a 6 ans lorsque son père meurt et que sa belle-mère le confie à l'Assistance publique. Son parcours ressemble aux deux précédents : plusieurs fugues le conduisent à l'âge de 16 ans à Mettray, où il ne restera que quelques mois. En effet, une provocation verbale de sa part entraîne de violentes représailles de la part d'un gardien. Un camarade lui prête main-forte. Ils s'évadent, mais sont rapidement repris par les gendarmes. Le Dano sera alors placé à la colonie agricole de Saint-Hilaire. Le désir de dénoncer les maltraitances et les injustices de l'institution est présent chez ces deux témoins, en particulier chez Jean-Guy Le Dano pour qui cette prise de parole constitue un véritable pamphlet. Les trois ouvrages ciblent une même période : les années 1920. De plus, il s'agit d'anciens colons issus tous trois de l'Assistance publique. Chacun évoque cependant une réalité singulière et manifeste une sensibilité, une mémoire attentives à des faits différents ; chacun choisit un mode d'écriture particulier et établit une relation différente à l'écriture. Parfois les témoignages convergent, parfois ils se révèlent absolument antinomiques. 
La question du corps des colons s'est imposée de façon évidente, puisqu'elle est au cour du récit genétien et de son ambivalence : d'une part, Genet montre et dénonce la violence terrible qui s'exerce au sein de la colonie sur ses pensionnaires, d'autre part, il offre une représentation féerique, poétique et idéalisée de ces mêmes colons. Nous aborderons tout d'abord le corps souffrant du colon, soumis à de durs travaux, à des privations de toutes sortes, aux différentes formes de violence qui s'exercent sur lui du fait de l'institution et de ses agents, mais aussi des autres colons. Puis, nous nous intéresserons au corps réinventé par les colons eux-mêmes. Il s'agira de montrer comment le corps devient le biais par lequel les colons, en effet, cherchent à se distinguer, à recouvrer une identité que l'institution cherche à masquer, à contraindre ; comment les colons créent des signes qui leur sont propres et qui leur permettent de se reconnaître ; comment le corps souffrant est réinventé par l'écriture de Genet, donnant naissance à un corps féerique. Il s'agira à la fois de dresser un portrait juste du corps des colons de Mettray et de rendre compte des représentations propres à chacun. Cet article n'entend pas se substituer à une étude historique ; il s'agit d'une approche littéraire, mais qui tente de se nourrir des apports de l'histoire. Cet éclairage et ce travail interdisciplinaire m'ont semblé intéressants pour ma propre recherche. Peut-être apportera-t-il un enrichissement à d'autres travaux.

\section{Un corps souffrant}

\subsection{Un corps emprisonné au cœur de l'Institution}

Les colons sont répartis en familles, chacune correspondant à un bâtiment. La répartition ne se fait pas nécessairement par âge, mais aussi par taille, ${ }^{(8)} \mathrm{ce}$ qui pose des problèmes d'abus et de violences des plus forts sur les plus faibles. Chaque famille se trouve sous l'autorité d'un colon, appelé prévôt ou frère aîné, qui seconde le gardien responsable, dit chef de famille. Les colons sont de ce fait observés, surveillés à tout moment de la journée par un personnel peu formé à l'éducation et souvent issu de l'Administration pénitentiaire. Mettray dispose d'une dizaine de cellules, où l'on place les colons en fonction de la faute commise. On y dort sur une paillasse, parfois privé de lumière, toujours privé de communication avec l'extérieur. Ainsi, malgré son cadre agréable, la colonie a tout d'une véritable prison.
165 p. ; Jean-Guy Le

Dano, La Mouscaille, Paris,

Flammarion, 1973, $330 \mathrm{p}$.

(7) Jean-Guy Le Dano, op. cit., p. 1.

(8) Les différents témoignages sont contradictoires sur ce point : Genet affirme que la répartition est faite par taille, Raoul Léger par âge, Jean-Guy Le Dano selon l'un ou l'autre critère... 
(9) Jean Genet, op. cit., p. 147. Voir aussi Raoul Léger, op. cit., p. 38-39 et Jean-Guy Le Dano, $o p$. cit., p. 23-24.

(10) Jean Genet, $o p$. cit., p. 326.

(11) Ibid., p. 45.

(12) Jean-Guy Le Dano, op. cit., p. 18, p. 20.

(13) Raoul Léger, op. cit., p. $55:$ «[.. ] sur les sept cent vingt repas du midi et du soir, il y était bien servi six cent soixante-dix fois des haricots blancs ou rouges, rarement du riz et quelques fois des lentilles, où il fallait faire très attention si l'on ne voulait pas se casser les dents, car il y avait pas mal de petits cailloux. "
Une discipline quasi militaire est imposée aux corps d'enfants parfois très jeunes et souvent en mauvaise santé. La vie est rythmée par le son du clairon et les ordres des surveillants. Les rituels du quotidien ne laissent aucune place au hasard, le jeu est quasiment banni. Les colons bénéficient d'une heure et demie de récréation par jour. Les trois auteurs restituent avec une précision terrible les différentes étapes de la journée du colon : le réveil et le coucher, les corvées au réfectoire, les déplacements, les heures de travail... Le rituel du coucher est particulièrement présent à leur mémoire :

"Voici la manœuvre du dortoir : quand tous les colons de la famille sont montés, le chef de famille ferme la porte à clé et la séance commence. Chaque colon, le dos au mur, se tient à sa place, sur chacun des grands côtés du dortoir. Le frère aîné crie "Silence" et les enfants s'immobilisent. "Enlevez les sabots", ils quittent leurs sabots et les placent sur une ligne très droite, à deux mètres en face d'eux. "À genoux”, crie le frère aîné. Les colons s'agenouillent devant les sabots vides de pieds, mais qui fument. "Prière." [...] "Enlevez les tringles." Ils soulèvent les grosses poutrelles accrochées au mur et, au commandement, ils viendront en placer le bout dans des encoches taillées dans des poutres verticales disposées là à cet effet. [...] On "déploie les hamacs", on "fait son lit", "plie ses effets" [...] " (9)

La majeure partie de la journée est vouée au travail, soit dans des ateliers : brosserie, cordonnerie, menuiserie, saboterie..., soit dans les champs ou la carrière. Le dimanche, seul jour de relâche, voit le loisir lui-même régi par des contraintes inépuisables : musique, exercices de natation, entraînement de l'équipe des sapeurs-pompiers... ; tout est cadré, ordonné, et bien sûr étroitement surveillé. En plus de ces activités, de nombreuses corvées sont réservées aux colons. Mais l'égalité n'est pas de mise : tout revient aux plus faibles, dits " cloches ", et aux nouveaux venus : cuisine, vaisselle, nettoyage des tables et du réfectoire, des toilettes..., les « durs » usant de leurs privilèges et de leur force... avec l'aval implicite des surveillants.

\subsection{Des conditions de vie déplorables}

L'alimentation des colons est insuffisante, déséquilibrée. Chez Genet, la nourriture est quasiment absente du récit. Il fait brièvement allusion à un déjeuner fait de «soupe " et de " pain noir ». ${ }^{(10)}$ Seule est évoquée la " faim 
d'enfants ", qui serait une " gloutonnerie naturelle ", ${ }^{(1)}$ moins féroce que celle qui ronge les prisonniers adultes. À son arrivée à la colonie, Jean-Guy Le Dano passe plusieurs jours en cellule d'isolement : on le nourrit uniquement d'une sorte de soupe de lentilles ou de pois cassés. ${ }^{(12)}$ Quant à Raoul Léger, s'il reconnaît le peu de variété des menus, ${ }^{(13)}$ il affirme manger à sa faim. ${ }^{(14)} \mathrm{La}$ " soupe du matin " est composée de " quelques ronds de carottes, d'oignons, le reste du pain ". ${ }^{(15)}$ Il affirme qu'on ne mange de la viande qu'une fois par an, le 14 juillet. ${ }^{(16)}$ Mais, au quartier, les rations sont encore réduites : une soupe le matin, " un morceau de pain à midi et un le soir ; le quart d'eau : un le midi, un le soir ", ${ }^{(17)}$ avec " une gamelle de haricots " tous les quatre jours. ${ }^{(18)}$ Cette nourriture entraîne des "maux de ventre et problèmes gastriques ". ${ }^{(19)}$ Les témoignages, comme les archives de Mettray, explique Éric Pierre, ne permettent pas de rendre compte des autres maladies récurrentes des colons. Ils souffrent sévèrement du froid en hiver, n'ayant pas de chaussettes. Il semble que des cas de décès des suites d'une tuberculose aient été relevés, avec un fort accroissement de la maladie à la fin des années 1920. ${ }^{(20)}$ Genet parle des " maigres corps " de ces " quatre cents enfants ». ${ }^{(21)}$ Raoul Léger évoque longuement son calvaire lors de son arrivée à la colonie : il souffre atrocement pendant un mois, devant porter des sabots à brides, à même la peau. Les pieds en sang, bientôt infectés, il doit se rendre à l'infirmerie. Ces " galoches " font partie de l'uniforme imposé, calqué sur un curieux mélange entre les vêtements des marins et ceux des prisonniers ou des bagnards.

En outre, l'hygiène est minimaliste : les colons n'ont accès au savon que deux fois par semaine, ils se lavent le visage dans des lavabos collectifs, constitués d'une "grande rigole en zinc de trente centimètres de large et vingt de profondeur " au fond du dortoir, avec " au-dessus un tuyau d'eau, percé de petits trous ". (22) Les toilettes sont ouvertes et l'on s'y rend par groupes de quatre ; soit une toilette pour dix colons environ. La longue et minutieuse description des " chiottes" manifeste le peu de respect des dirigeants pour la dignité des colons : on s'y rend à heure dite, en rangs par quatre, les murs témoignent de l'absence de papier. "C'était un vulgaire trou donnant sur une fosse, avec une demi-porte, aucun papier, d'où les murs pleins de virgules de merde, faites avec le majeur de la main, si bien qu'après avoir fait la queue aux chiottes, il y avait la queue au robinet. " ${ }^{(23)}$ On retrouve la même des-
(14) Ibid. p. 36.

(15) Ibid. p. 41.

(16) Ibid. p. 80.

(17) Ibid. p. 62.

(18) Ibid. p. 63.

(19) Éric Pierre,

«Mettray dans les années 1920. Une période noire ", in Raoul Léger, La colonie agricole et pénitentiaire de Mettray. Souvenirs d'un colon I922I927, Paris, L'Harmattan, 1997, p. 149.

(20) Ibid. p. 49.

(21) Jean Genet, $o p$. cit., p. 329.

(22) Raoul Léger, $o p$. cit., p. 40 .

(23) Ibid. p. 40.

(24) Jean Genet, op. cit., p. 222.

(25) Jean-Guy Le Dano, op. cit., p. 29.

(26) Éric Pierre, op. cit., p. 149. 
(27) Raoul Léger, $o p$. cit., p. 61.

(28) Jean Genet, op. cit., p. 319 : « M. Bienveau était le grand maître du quartier. Sa bouche était fermée sur des dents serrées, et l'on ne voyait pas son regard noir, derrière des binocles. Il portait, été comme hiver, un extraordinaire canotier de paille jaune entouré d'un large ruban de faille bleu ciel. [...] Pour l'été, il avait inventé de faire apporter une bassine pleine d'eau fraîche et il nous regardait mourir au soleil quand lui-même prenait des bains de pieds qui duraient trois heures. »

(29) Jean-Guy Le Dano, op. cit., p. 17-19.

(30) Jean Genet, $o p$. cit., p. 314. Le piquet consiste en « deux heures chaque jour de mouvements fatigants, exécutés dans la cour du quartier, en place de récréation". cription chez Genet. ${ }^{(24)}$ Jean-Guy Le Dano relève que, dans les cellules d'isolement, il n'y a pas de tinette, mais un caniveau qui passe de cellule en cellule, provoquant l'inondation fréquente de la dernière... ${ }^{(25)}$ On note enfin de nombreux accidents du travail, mais aussi de fréquentes automutilations de colons désireux de passer quelques jours à l'infirmerie. ${ }^{(26)}$

\subsection{Violences perpétrées par les gardiens}

Deux gardiens sont évoqués chez Genet : le violent Guépin et M. Bienveau - sans doute le Biavaux de Raoul Léger, " chef du Quartier ", "l'être le plus ignoble de tous les gardiens de la colonie " ${ }^{(27)}$ - dont Genet rédige un portrait mordant. ${ }^{(28)}$ Raoul Léger présente le surveillant Guépin comme quelqu'un de ferme, mais de juste. Plusieurs passages témoignent de son humanité. JeanGuy Le Dano signale qu'il a été accueilli par les cris d'un certain Kalifia, puis par les coups de pied et les coups de poing d'un autre gardien qui le laissera inanimé dans sa cellule. ${ }^{(29)}$ De façon générale, les surveillants, que les colons appellent " gaffes " ou " gâfes " comme dans les prisons, sont présentés comme des êtres frustes et violents. Tout manquement est puni par des mises au piquet, ${ }^{(30)}$ des privations de nourriture, des mises en cellule en fonction du degré de la faute. ${ }^{(31)}$ Le jeune Raoul Léger va se distinguer par sa forte fréquentation des cellules du quartier, où il cumule des peines de quinze, trente jours... Les colons punis doivent arpenter, toute la journée durant, une cour où seul le bruit de leurs sabots résonne. ${ }^{(32)}$

Les passages à tabac ne semblent pas rares, au point que le bruit court que certains colons n'auraient pas survécu aux violences des surveillants. Jean-Guy Le Dano affirme avoir assisté à la mort de l'un de ses camarades battu par un gardien, cas qui fut signalé comme un décès dû à la tuberculose !(33) Il raconte également qu'un " frère aîné n'a rien trouvé de mieux que de tuer, en se battant, un colon d'un seul coup de pied dans la tête ». ${ }^{(34)}$ Cet événement aurait été à l'origine de la fermeture de la colonie. Fait réel ou événement inventé en vue de renforcer encore l'argumentaire contre ces bagnes d'enfants? Genet évoque, quant à lui, des "gâfes " fouettant jusqu'au sang ou frappant à coups de trique des colons " [parce] qu'ils murmuraient trop bas ", ${ }^{(35)}$ mais surtout la mort d'un colon assassiné après une tentative d'évasion, retrouvé dans un bosquet, " percé de quatorze coups de tranchet ».(36) Pour l'un comme pour 
l'autre, on assassine des enfants au vu et au su de tous, et personne ne dénonce, personne n'agit. Raoul Léger reconnaît la brutalité de certains gardiens, il eut à en souffrir lui-même, mais il déclare que "de 1922 à 1927, il n’y a eu aucun colon tué par des gardiens, je dis bien aucun : des brutalités, oui ; mais ils savaient jusqu'où ils pouvaient aller ! " ${ }^{(37)}$ Ce qui ne l'empêche pas d'envisager un gardien violent comme un assassin en puissance, après avoir assisté au passage à tabac d'un colon très affaibli - car soumis au piquet et privé d'eau - et laissé pour mort. ${ }^{(38)}$ Quoi qu'il en soit, la violence des surveillants dépasse de loin les rappels à l'ordre exigés. Certains ne savent pas se maîtriser, d'autres y trouvent une forme de divertissement.

Raoul Léger conteste ${ }^{(39)}$ également la véracité du passage où Genet et son " marle » Villeroy attaquent le surveillant Guépin et se battent contre lui. ${ }^{\left({ }^{(0)}\right.}$ Les deux colons victorieux n'auraient écopé que d'un mois de quartier. Peine bien faible, au vu de leur action d'éclat. En guise de comparaison, notons que Jean-Guy Le Dano partira pour Saint-Hilaire, suite à sa tentative d'évasion et aux quelques larcins qu'il commit pendant sa courte cavale. Il nous semble que cette mise en scène montre en premier lieu le changement de statut du chétif Genet qui, comme Raoul Léger, devra s'imposer face à des colons plus forts. Battre un gardien, n'est-ce pas la preuve ultime de sa valeur, de sa virilité ? En outre, cette scène de fiction symbolise la victoire des colons sur leurs tortionnaires. Cette victoire sur le papier a tout d'une revanche.

\subsection{Violences entre colons}

L’organisation interne des colons imite en tout point celle de leurs aînés, prisonniers des maisons d'arrêt. Genet décrit avec force détails le système hiérarchisé, voire féodal, mis en place par les colons pour gérer leur communauté. Dans chaque famille, il y a trois catégories : les " durs » ou " marles », les " vautours » et les " cloches ». Les premiers sont les mâles, forts, craints, respectés, les seconds sont leurs mignons, leurs favoris, les derniers sont les esclaves, les souffre-douleur du reste de la colonie. Genet prétend que la famille Jeanne d'Arc était uniquement composée de "vautours des marles des autres familles ", ${ }^{(41)} \mathrm{car}$ elle regroupait les plus petits parmi les colons. Cette société marginale est fondée sur des rapports de force et sur les rôles sexuels qui en découlent. Chacun est tenu de rester à sa place, de tenir son rang, sous peine de dures représailles.

(31) Henri Gaillac, Les maisons de correction I830I945, Paris, éditions Cujas, 1991, p. 88 ; Jacques Bourquin, "Mettray dans les années 1920. Une période noire. ", in Léger Raoul, La colonie agricole et pénitentiaire de Mettray. Souvenirs d'un colon 19221927, Paris, L'Harmattan, 1997, p. 125.

(32) Raoul Léger, op. cit., p. 31 et p. 62.

(33) Jean-Guy Le Dano, op. cit., p. 32.

(34) Ibid., p. 31.

(35) Jean Genet, op. cit., p. 331-332.

(36) Ibid., p. 326.

(37) Raoul Léger, op. cit., p. 108.

(38) Raoul Léger, op. cit., p. 63-64.

(39) Ibid., p. 108. 
(40) Jean Genet, op. cit., p. 155-160.

(41) Jean Genet, op. cit., p. 143.

(42) Ibid., p. 216-217.

(43) Raoul Léger, op. cit., p. 56.

(44) Jean Genet, op. cit., p. 217.

(45) Jean Genet, op. cit., p. 201-202 et p. 344-346.

(46) Ibid., p. 315-317.

(47) Jean Genet, op. cit., p. 321-322.

(48) Jean-Guy Le Dano, op. cit., p. 88-89 : "Les vieilles taules pourront être rasées et remplacées par des modernes, les régimes améliorés, les gaffes déguisés en bonnes sœurs, rien ne sera réglé tant que ce problème humain [séparation des hommes et des femmes
L'entrée dans la colonie est une épreuve difficile, où le nouveau venu est testé par les autres colons et parfois véritablement persécuté. Les corvées humiliantes lui sont réservées. Si le colon se distingue, il appartient aux " marles », mais s’il manifeste un signe de faiblesse, il a tout intérêt à trouver rapidement un protecteur. Ainsi Raoul Léger va, dans un premier temps, souffrir des railleries et des mauvais traitements de son frère aîné, Jégouzo. Puis, grâce à deux camarades, il va apprendre à se défendre et il se libérera de son bourreau. S’il n'est pas assez beau ou séduisant, le colon intégrera les "clodos ", qui " étaient le peuple noir et laid, chétif et rampant sans quoi le patricien n'existe pas ", menant une "vie d'esclaves ». ${ }^{(42)}$ Genet n'hésite pas cependant à comparer ces relations à celles d'un chevalier avec sa dame, avec un vassal. Les liens qui se tissent semblent indissolubles ; les colons, malgré toutes ces horreurs, semblent solidaires et fidèles à leur code de l'honneur. Sans recourir comme Genet à des images ou à une tradition littéraire, Raoul Léger et Jean-Guy Le Dano soulignent à plusieurs reprises l'importance et la force des amitiés tissées entre colons, seul véritable soutien dans cet univers de violence.

Tout se règle par une bagarre. "À Mettray, s'il y avait bagarre, bien souvent le gaffe n'intervenait qu'après que les deux mecs s'en soient mis plein la pipe. Car les bagarres entre colons étaient très violentes. Tous les coups étaient permis, même à terre, et les coups de pieds, avec les sabots ferrés, faisaient très mal. " ${ }^{(43)}$ Lorganisation fortement hiérarchisée des colons conduit à un règne de la force et de la violence sous toutes ses formes. Les " marles " se battent entre eux pour assurer leur prestige, prouver leur valeur. Ils maltraitent les « cloches » pour affirmer leur statut privilégié, et y trouvent de plus du plaisir et un divertissement. Passages à tabac, humiliations, viols..., rien ne leur est épargné. Les scènes écrites par Genet sont parfois à la limite de l'insoutenable.

Entrons-nous déjà dans la fiction ou sommes-nous encore les témoins de souvenirs réels lorsque Genet évoque Larochedieu, obligé de se déshabiller dans la cour pour montrer ses traces de coups ? ${ }^{(44)}$ De même lorsqu'il décrit la scène où les " marles" assemblés crachent sur un colon à tour de rôle ${ }^{(45)}$ ou lorsqu'il raconte les "jeux cruels " des " marles " ? (46) Sa description d'un colon soumis à des viols collectifs se passe de commentaires : "Pour Winter, la beauté fut un coup dur. Les durs s'éprirent de lui et il eut la souffrance d'être par douze bites enfilé, et la honte de l'être presque publiquement. [...] 
Winter se coupa les cils pour être moins beau. Il changea de famille et devint cloche. Mais je l'ai vu se débarbouiller de ses larmes après avoir été nettoyé au foutre par une douzaine de marles. " ${ }^{(47)}$

Ce système de castes, pourrait-on dire, ainsi que la promiscuité des adolescents entraînent une sexualité brutale et peu épanouissante, qui rappelle celle des condamnés adultes, dénoncée fortement par Jean-Guy Le Dano. ${ }^{(48)}$ Ici encore les témoignages divergent. Pour Raoul Léger, il n'y a aucune relation sexuelle au sein de la colonie : Genet aurait "romancé comme il aurait voulu que ça se passe, pour satisfaire ses fantasmes d'homosexuel. " (49) Il raconte pourtant s'être battu avec un colon qui lui faisait des œillades. ${ }^{(50)}$ Le Dano est plus explicite : "À Mettray, la pédérastie est le sport le plus usité : la moitié des colons se fait joyeusement enjamber par l'autre moitié. " (51) Genet, non seulement affirme l'existence de ces pratiques, mais il semble dire qu'elles sont systématiques. L'homosexualité serait, selon lui, pour la plupart des jeunes gens, une sexualité de substitution ou une " homosexualité passagère ": "Puisqu'il n'y avait pas de filles, il fallait bien. " ${ }^{(52)}$ Genet avoue ses amours, il s'en vante même. Les deux autres " témoins " réfutent toute relation ou désir homosexuels et ne souhaitent peut-être pas revenir sur de douloureux souvenirs d'humiliations et de tortures.

Les dirigeants de Mettray ont employé tous les moyens pour réformer les colons, leur inculquer une nouvelle moralité et un métier. Mais il semble que la réforme morale passe avant tout par une réforme complète du corps. Celui-ci est soumis à des conditions de vie extrêmes qui vont le modeler, l'endurcir. Il supporte des privations, des souffrances et des agressions quotidiennes. Le corps outil de travail n'est plus qu'un corps souffrant. L'institution et ses personnels outrepassent leurs objectifs et leurs devoirs en s'appropriant les corps des colons, en les maltraitant, mais aussi en refusant de les défendre contre leurs condisciples. Face à cette forme de déshumanisation et à cette multiplication de la violence, les colons tentent de trouver de rares espaces de liberté, d'individualité. Ils parviennent à reprendre possession de leurs corps opprimés et cassés et d'en faire, malgré tout, les étendards de leur singularité, en développant des signes qui leur sont propres. condamnés de l'autre sexe] ne sera pas franchement reconnu d'utilité ublique. " (p. 89).

(49) Raoul Léger, op. cit., p. 108.

(50) Ibid., p. $94-96$ et p. 98 .

(51) Jean-Guy Le Dano, op. cit., p. 24.

(52) Jean Genet « Entretien avec Hubert Fichte ", in L'ennemi déclaré, "Euvres complètes VI”, Paris, Gallimard, 1991, p. 170. 
(53) Jean Genet,

Miracle..., op. cit., p. $162-163$.

(54) Raoul Léger, op. cit., p. 68-70.

(55) Jean Genet, Miracle.., op. cit., p. 199.

(56) Ibid., p. 263-264.

(57) Raoul Léger, op. cit., p. 56.

(58) Ibid., p. 92.

(59) Ibid., p. 28 et 32.

(60) Ibid., p. 37.

(61) Jean-Guy Le Dano, op. cit., p. 21.

(62) Raoul Léger, op. cit., p. 55.

\section{Se réapproprier son corps}

\subsection{Attitudes corporelles et détournement de l'uniforme}

Pour signifier son identité, chaque catégorie de colons possède des attitudes spécifiques, ainsi que des attributs propres. Les « cloches » doivent porter leurs vêtements de la façon la plus simple possible et adopter une attitude soumise. Ils ne peuvent refuser les demandes des "marles ». Jean Genet explique comment il prit des allures de " dur ", après avoir appris à se battre, à garder les jambes écartées, à siffler. ${ }^{(53)}$ De la même façon, Raoul Léger raconte comment l'un de ses amis lui apprit à se battre et à ne se laisser dominer par personne. ${ }^{(54)}$ Un même colon peut cependant évoluer, passer d'une catégorie à une autre. Genet en serait la preuve, tout comme Raoul Léger. "Tous les marles furent la fiancée mystique de quelque dur ", ${ }^{(55)}$ affirme Genet. Villeroy obligera le narrateur à prendre un "vautour » à son tour pour montrer son pouvoir, son statut de futur « marle». ${ }^{(5)}$ Genet ne se présente cependant jamais comme une " cloche », il préfere à ce terme des périphrases ou des images qui masquent la réalité humiliante de ce statut. Il se dit la dame ou la courtisane d'un " marle ». Raoul Léger est plus direct : il affirme avoir souffert de cet état de victime des " marles ». ${ }^{(57)}$ Après plusieurs bagarres et plusieurs séjours au quartier, il a acquis le respect des autres colons, et en particulier celui des " marles ». " [Il nétait] plus la cloche qu' [il était] au début ". ${ }^{(58)}$

Les colons portent un uniforme rudimentaire qui rappelle le costume des bagnards : une blouse en toile blanc écru et un pantalon de couleur gris/bleu, des sabots, des bandes molletières, un grand chapeau de paille, plat, à large bord. ${ }^{(59)}$ Raoul Léger insiste sur cette ressemblance : les colons portent les mêmes vêtements que les bagnards, mais de plus, comme eux, ils travaillent dans une carrière, ils cassent des cailloux ! ${ }^{(60)}$ Jean-Guy Le Dano précise qu'ils portaient un " tricot rayé comme en portent les bagnards ", un " calot noir de soldat [et] une paire de bandes molletières de même teinte ».(61) Pour le dimanche, ils disposent d'une vareuse bleu marine avec des boutons de cuivre, d'un pantalon et d'un béret à pompon rouge. Cette tenue leur donne " un air de petits mariniers ". ${ }^{(62)}$ La contrainte s'exerce à plusieurs niveaux : tout d'abord rien ne les distingue les uns des autres a priori, si ce n'est leur numéro. Chaque colon dispose en effet d'un matricule qu'on retrouve sur ses vêtements. JeanGuy Le Dano signale qu'on peut l'effacer très facilement, ce qui explique les 
nombreux vols ou " échanges » de vêtements entre colons, malgré l'interdiction qui en est faite ; fait contesté par Raoul Léger. Ensuite, ces vêtements sont loin d'être agréables ou même décents, comme le prouve l'anecdote des sabots de Raoul Léger. Enfin, ils sont insuffisants en nombre et en qualité.

Mais les jeunes gens, copiant les prisonniers, leurs aînés, s'approprient et transforment les quelques rares éléments qui leur sont alloués. L'uniforme est habilement détourné pour laisser une place à la singularité des colons, ou du moins des plus forts. Les " marles ", en effet, cherchent à se différencier des autres colons. Pour ne pas être une "cloche ", il faut troquer ses vêtements contre "des effets moins caves ", ${ }^{(63)}$ recourir à une élégance de fortune. Ainsi la façon crâne dont ils portent leur béret, renforcé par un fil de fer glissé à l'intérieur du couvre-chef, marque leur supériorité. On les distingue encore par la fausse poche ajoutée aux pantalons, le travail des sabots dont on lime le bois pour les rendre plus fins et modifier leur forme. ${ }^{(64)}$ Certains portent le pantalon droit, le pli tombant bien net sur le pied. La disposition des bandes molletières doit mettre en valeur la force physique de son propriétaire : elles " devaient mouler un mollet solide, puissant, rendu plus imposant encore par le pantalon retroussé sous la bande ». ${ }^{(65)} \mathrm{La}$ couleur des blouses qu'on essaye de blanchir, la souplesse des pantalons et des blouses si rêches au départ sont autant de signes distinctifs et de moyens d'individualiser l'uniforme. Les " clops » et un briquet bricolé de toutes pièces complètent la tenue des jeunes " caïds ». ${ }^{(6)}$ Le portrait de Divers dans Miracle de la rose résume parfaitement cette élégance conquise de haute lutte. ${ }^{(67)}$ Ce colon retaille ses " frocs » pour qu'ils soient plus collants, il porte un poignet de force... Rien n'est précisé de la tenue des autres catégories de colons, mis à part le fait qu'il leur est strictement interdit de modifier leur apparence et de copier leurs "supérieurs ". De ce fait, ils devaient garder "[leurs] sabots aussi lourds, [leur] blouse aussi neuve, [leur] cravate aussi coupante " ${ }^{(68)}$ qu'à leur arrivée à Mettray.

\subsection{Tatouages}

Seul Genet décrit les tatouages des colons. Raoul Léger et Jean-Guy Le Dano n'en parlent pas. Raoul Léger évoque brièvement un tatouage fait par la suite à Calvi, mais il ne conteste pas les dires de Genet. Qu'il s'agisse d'une réalité ou d'un fait fictif, le tatouage a une valeur symbolique importante
(63) Jean Genet,

Miracle.., op. cit., p. 170.

(64) Ibid., p. 170.

(65) Jean Genet,

Miracle..., op. cit., p. 170.

(66) Raoul Léger, op.

cit., p. 81 .

(67) Jean Genet,

Miracle..., op. cit., p. 323324.

(68) Ibid., p. 170. 
(69) Jean Genet,

Miracle..., op. cit., p. 9-10 :

Le détenu de la centrale

de Fontevrault constitue

« la forme même du

futur » des colons

de Mettray. «[Le colon]

savait que sa forme défini-

tive résidait derrière

eux, et que ce puni de

trente berges était l'extrê-

me réalisation de lui-

même, le dernier avatar

que la mort fixerait. »

(70) Ibid., p. 194.

(71) Ibid., p. 196.

(72) Ibid., p. 200.

(73) Ibid., p. 193.

(74) Ibid., p. 197.

(75) Ibid., p. 196.

(76) Raoul Léger, op. cit., p. 109.

(77) Albert Dichy et Pascal Fouché, op. cit., p. 127.

(78) Raoul Léger, op. cit., p. 108. dans le récit et il rattache les colons aux prisonniers adultes qu'ils deviendront pour la plupart. ${ }^{(69)}$ Pour Genet, les tatouages constituent l'héraldique du monde des colons, le signe de leur dignité, de leur noblesse propre. Peut-on y voir un rappel des anciennes marques des condamnés et des forçats ? Les colons substituent la pensée à la fleur de lys, affichent leurs propres signes, transformant marque d'infamie en marque de noblesse. Ils dessinent sur leurs corps les signes de leur liberté, de leur communauté opposée à une société et à des règles que tentent de leur imposer la colonie et ses représentants. Le signe distinctif des " marles" - en plus des accessoires et vêtements - est le tatouage. Les colons copient en cela les criminels des maisons d'arrêt. On n'accède à l'honneur d'être tatoué qu'avec l'autorisation des "caïds » les plus importants : ainsi «Villeroy, le marle de Genet, refusait à Beauvais l'autorisation de l'Aigle ». ${ }^{(70)}$ Genet affirme avoir vu " des gars tatoués de l'Aigle, de la Frégate, de l'Ancre de Marine, du Serpent, de la Pensée, des Étoiles, de la Lune et du Soleil ». ${ }^{.71)}$ Genet se dit particulièrement fasciné par un colon au corps entièrement recouvert de tatouages : Gaveille qu'il associe à « la forme visible d'un mâle - une fleur mâle ". ${ }^{\text {(72) }}$ Signe de reconnaissance et de complicité entre les colons, le tatouage est aussi un signe chargé d'érotisme.

Genet dépasse la simple évocation de souvenirs pour réécrire le passé et lui donner une véritable grandeur. Les colons ne sont plus seulement des enfants meurtris, mais les chevaliers d'un royaume fictif. Les colons forment « l'Ordre des Tatouages ", ${ }^{(73)}$ symbolisé par une pensée autour d'une banderole. "C'est pour que la fleur et la banderole reposent dans un cadre dignes d'elles [qu'ils sétaient] fait tatouer d'ornements qui gagnaient tout le corps. " ${ }^{(74)}$ Les tatouages constituent les ornements d'un corps devenu étendard, les blasons d'une " chevalerie nouvelle ", " les parchemins " ${ }^{(75)}$ d'une histoire en marge de l'Histoire reconnue. Le corps porte la marque et la mémoire de ce qu'il a vécu, avec fierté. Le tatouage, marque volontaire sur et dans le corps, s'oppose aux traces de coups et aux cicatrices imposées par les " gâfes".

\subsection{Réinvention du corps par l'écriture}

Raoul Léger conteste plusieurs passages de Miracle de la rose qui lui paraissent une " affabulation " et du "vrai délire ». ${ }^{(76)}$ Il cite ainsi la bagarre avec le surveillant Guépin déjà évoquée, le mariage des colons à l'église, la mort d'un 
colon brutalisé et la révolte générale. La véracité de ces différents passages peut certes être mise en question, mais Raoul Léger ne semble considérer Miracle de la rose que comme un témoignage, totalement irrecevable au demeurant. La dimension littéraire, donc fictive, du livre de Genet semble lui échapper complètement. Tout à sa propre démarche, peut-être ne peut-il pas accepter de voir un passé trop douloureux et trop longtemps tu, transformé, déformé, voire trahi par le recours à la fiction. Ce qui pourrait expliquer pourquoi il refuse de croire que Genet a été lui aussi un colon. L'homosexualité affirmée de ce dernier et sa vision des amours masculines à Mettray le choquent également. Cette lecture de Genet par Raoul Léger est d'autant plus intéressante qu'elle montre la limite des rapprochements possibles entre les trois ouvrages. Seul Genet ajoute à son témoignage une recréation du passé. On peut considérer, avec Albert Dichy et Pascal Fouché, que « les éléments factuels qui relèvent du mode narratif participent d'une très savante ambiguïté : ils sont alternativement, si on les place en regard de l'instance biographique, vrais ou faux et sans être jamais, tout à fait, l'un ou l'autre ». ${ }^{(77)}$ Peut-être pourrait-on également contester des faits évoqués par les deux autres témoins. Mais leur volonté est affichée : dire la vérité de ce qu’ils ont vécu. Chez Genet, rien de tel, la dénonciation et la description pures sont toujours associées à un récit dont on ne sait s'il repose ou non sur un fait réel ou inventé.

La représentation du corps des colons, me semble-t-il, oscille entre ces deux pôles. Genet, en effet, ne cesse de louer la beauté des colons. Mais peut-on se contenter de juger qu'il en profite pour "satisfaire ses fantasmes d'homosexuel » ? ${ }^{(78)}$ Genet a été sensible à cet aspect, en plus d'avoir évoqué les différents aspects décrits par les deux autres témoins. Ce chant participe d'un désir d'idéaliser les colons au sein de cet Enfer où pourtant il fut « véritablement heureux $"{ }^{(79)}$ Le paradoxe de son récit réside dans cette ambivalence du souvenir et de la réalité vécue : l'horreur peut côtoyer le bonheur, l'Enfer peut aussi se révéler être le Paradis. Les trois témoins furent frappés par l'apparence trompeuse et surprenante de la colonie : il s'agit d'une prison gardée par des fleurs. ${ }^{\left({ }^{80)}\right.}$ C'est " un jardin délicieux, carré, orné d'arbustes et d'une vasque ", ${ }^{\left({ }^{(1)}\right.}$ "l'endroit le plus beau de la plus belle Touraine ". ${ }^{(82)}$ Genet souligne la perversité et l'hypocrisie ${ }^{(83)}$ de ce cadre bucolique. Là où il se distingue des autres témoins, c'est en présentant la colonie comme un lieu typique de contes de
(79) Ibid., p. 170.

(80) Raoul Léger, op. cit., p. 27 ; Jean-Guy Le Dano, op. cit., p. 22 ; Jean Genet, op. cit., p. 13 et 20 .

(81) Jean Genet, Miracle..., op. cit., p. 13.

(82) Ibid., p. 20.

(83) Jean Genet,

«Entretien avec Antoine Bourseiller " (1982), in L'Ennemi déclaré, op. cit., p. 223 : «Une des finesses des inventeurs de la colonie de Mettray, c'est d'avoir su ne pas mettre de muraille. [...] Il est beaucoup plus difficile de s'évader quand il s'agit simplement de traverser un parterre de fleurs que de traverser une muraille. [...] Ils avaient inventé cette poésie, ils nous avaient terrorisés avec des pensées, des œillets, des lauriers, etc. " 
(84) Jean Genet, Miracle..., op. cit., p. $140-141$

(85) Ibid., p. 174.

(86) Ibid., p. 323.

(87) Ibid., p. 324-325.

(88) Ibid., p. 223.

(89) Ibid., p. 155 :

"Cette brute pour m'aimer se faisait craintive. Elle m'appelait Sapotille. Un soir même, il appela sa verge : « ma brutale », et la mienne « ta banette». Ces noms leur restèrent. Je sais à présent que, sans les prononcer, nous échangions les plus belles répliques amoureuses dans le style enchanté de Roméo et Juliette. Notre amour chantait dans cette désespérante baraque. "

(90) Ibid., p. 155.

(91) Ibid., p. 196.

(92) Ibid., p. 195-196. fées : d'une part, les fleurs y sont vivantes, elles sont les véritables gardiennes des colons et, d'autre part, les colons sont décrits comme des fleurs humaines. ${ }^{(84)}$ On quitte le récit réaliste et le témoignage pour basculer véritablement dans la féerie. De nombreux portraits des colons illustrent cette esthétique, alliant érotisme et merveilleux. Le personnage de Divers est ainsi présenté comme un séduisant joueur de tambour, au corps mis en valeur par l'uniforme. Il est si beau que " [son] tambour l'applaudissait ». ${ }^{(85)}$ Il fut même "pendant trois années le garçon le plus beau de la colonie qui contenait une centaine d'adolescents splendides $"{ }^{\left({ }^{(8)}\right)} \mathrm{La}$ description quitte le simple souvenir lorsque Genet avoue avoir rêvé d'une étreinte avec lui et s'être imaginé Ganymède face à l'aigle divin. ${ }^{(87)}$ Dès lors, le corps n'est plus seulement le corps décrit dans sa souffrance, mais un corps digne des blasons, un corps parfait et rêvé.

Dans son récit, Genet chante les amours qu'il a vécues et déclare qu’il " aime Mettray, ce paradis au cour de la Touraine royale, toute parcourue de petites veuves de 14 ou 16 ans, et de mâles frappés par la foudre aux plus beaux endroits $"{ }^{\left({ }^{(8)}\right)} \mathrm{La}$ description des relations sexuelles associe crudité et poésie. La première étreinte du narrateur et de Villeroy dans un hamac a tout d'un pugilat à première vue, or ce qui est souligné pourtant, c'est le plaisir partagé et une forme de tendresse. ${ }^{(89)}$ Notons que c'est l'un des rares passages où la description du corps est abandonnée au profit de celle du visage " contracté par la passion ", " phosphorescent ». ${ }^{(90)}$ Certaines scènes appartiennent au fantasme, à la « féerie " ${ }^{(91)}$ pure : ainsi la secrète scène de noces ${ }^{(92)}$ entre Genet et Divers, escortés par toute la famille B., comme autant de « colombes ou colons ". La poésie l'emporte dans ce passage où tout est immatériel, des " attributs des noces " aux pas et aux étreintes des invités.

C'est bel et bien par le travail du corps que l'institution tente de réformer les corps et les esprits des colons, qu'elle tente de leur imprimer sa marque au fer rouge. Vêtus comme des bagnards ou des militaires, assommés de travaux et de corvées, soumis à de mauvais traitements en permanence, les colons que décrivent nos trois témoins possèdent un corps meurtri et en mauvaise santé. Or, c'est à travers leurs corps que les colons développent leur espace de résistance et de liberté, à défaut de pouvoir se rebeller et de prendre la parole. Ils détournent l'uniforme imposé, se créent leurs propres codes de reconnaissance. Jean Genet, en mêlant la fiction à ses souvenirs, propose une vision 
féerique du corps des colons. Les témoignages permettent de dénoncer les abus vécus et de préserver la mémoire des anciens colons. La part fictive de Miracle de la rose offre un hommage poétique à ces corps magnifiés par l'écriture, comme vengés de toutes leurs souffrances.

\section{Bibliographie}

\section{Euvres étudiées}

Genet (Jean), Miracle de la rose (1946), Paris, Folio, Gallimard, 1977, 376 p. Le Dano (Jean-Guy), La Mouscaille, Paris, Flammarion, 1973, 330 p.

Léger (Raoul), La colonie agricole et pénitentiaire de Mettray. Souvenirs d'un colon I922-I927, Paris, L'Harmattan, 1997, 165 p.

\section{Autres œuvres de Jean Genet}

L'enfant criminel, in "Euvres complètes V", Paris, Gallimard, 1979, p. 377-393.

"Entretien avec Antoine Bourseiller» (1982) in L'ennemi déclaré, "Euvres complètes VI”, Paris, Gallimard, 1991, p. 217-226.

"Entretien avec Hubert Fichte» (1975) in L'ennemi déclaré, "CEuvres complètes VI”, Paris, Gallimard, 1991, p. 141-176.

\section{Biographies de Jean Genet}

Dichy (Albert), Fouché (Patrice), Jean Genet. Essai de chronologie I9I0-I944, Bibliothèque Jean Genet, Bibliothèque de littérature française contemporaine de l'université Paris 7, Paris, 1988, 294 p.

Moraly (E.), Jean Genet, la vie écrite, Paris, éd. de la Différence, 1988, 353 p.

White (Edmund), Jean Genet, (traduit par P. Delamare), Paris, NRF, Gallimard, 1993, 686 p.

\section{Ouvrages critiques consacrés à Jean Genet}

El Basri (Aïcha), L’imaginaire carcéral de Jean Genet, Paris, L'Harmattan, 194 p. Éribon (Didier), Une morale du minoritaire. Variations sur un thème de Jean Genet, Paris, Fayard, 2001, 342 p.

Jablonka (Ivan), Les vérités inavouables de Jean Genet, Paris, Seuil, 2004, 434 p. 


\section{Ouvrages et articles consacrés ou partiellement consacrés à la question des « bagnes d'enfants"}

Bourquin (Jacques), "Le Mettray des origines », in Léger (Raoul), La colonie agricole et pénitentiaire de Mettray. Souvenirs d'un colon 1922-I927, Paris, L'Harmattan, 1997, p. 113-126.

Foucault (Michel), Surveiller et punir, Paris, Gallimard, 1975, 463 p.

Gaillac (Henri), Les maisons de correction I830-1945, Paris, éd. Cujas, 1991, $360 \mathrm{p}$.

Guyotat (Régis), "Genet à Mettray ", Le Monde, 3 octobre 1992.

Pierre (Éric), " Mettray dans les années 1920. Une période noire. ", in Léger (Raoul), La colonie agricole et pénitentiaire de Mettray. Souvenirs d'un colon I922-I927, Paris, L'Harmattan, 1997, p. 127-151.

Roumajon (Yves), Enfants perdus, enfants punis. Histoire de la jeunesse délinquante en France : huit siècles de controverse, Paris, Robert Laffont, 1989, $352 \mathrm{p}$.

«Dossier : 1839-1937. La colonie pénitentiaire agricole de Mettray. Un siècle d'histoire de l'éducation des enfants assistés et condamnés ", Le Magazine de la Touraine, octobre 1983, nº 3, p. 3-22.

\section{Divers}

Bauche (Henri), Le langage populaire. Grammaire, syntaxe et dictionnaire du français tel qu'on le parle dans le peuple de Paris, avec tous les termes d'argot usuel, Paris, Payot, 1916, (rééd. 1920).

Lacassagne (Jean), L'argot du milieu, Paris, Albin Michel, 1935. 